\title{
Recurrent syncope and hypocalcaemic cardiomyopathy as manifestations of Fahr's syndrome
}

Marlena Broncel, Marzena Koziróg, Justyna Zabielska, Adam R. Poliwczak

Department of Internal Diseases and Clinical Pharmacology, Medical University of Lodz, Poland

Submitted: 19 December 2008

Accepted: 5 March 2009

Arch Med Sci 2010; 6, 1: 117-121

DOI 10.5114/aoms.2010.13518

Copyright $\odot 2010$ Termedia \& Banach

\begin{abstract}
In our report we would like to present a case of a 60-year-old patient with epileptic seizures, affective disturbances, only mild neurocognitive disorders and cardiomyopathy. A female patient was taken to the internal ward with a tentative diagnosis of recurrent syncope. Laboratory results disclosed severe hypocalcaemia, hypoparathyroidism, and hypothyroidism. An echocardiogram revealed left ventricle systolic dysfunction. Computed tomography revealed massive intracranial calcifications typical for Fahr's syndrome. Our patient demonstrated only mild neurological and psychiatric symptoms, but developed hypocalcaemic heart failure. It is possible that some cases of Fahr's syndrome remain undiscovered, particularly patients taken to internal wards with mild neurological or psychiatric signs.
\end{abstract}

Key words: Fahr's syndrome, cardiomyopathy, attacks of unconsciousness.

\section{Introduction}

Fahr's syndrome, also called bilateral striatopallidodentate calcinosis (BSPDC), is a disease characterized by symmetric, non-atherosclerotic calcification of the basal ganglia. Both familial and sporadic cases have been previously reported. A locus on chromosome $14 q$ has been identified as associated with BSPDC [1]. Familial type of the syndrome has been previously described [2]. However, it has also been reported that BSPDC is not linked to the IBGC1 locus on chromosome 14q [3]. Calcium deposits are the major element responsible for the radiological appearance of the disease. Hypoparathyroidism and disturbance of calcium metabolism can be responsible for BSPDC development, but cases of patients with normal parathormone levels have also been reported [4]. Pseudohypoparathyroidism associated with production of biologically inactive form of parathormone can also be connected with BSPDC pathology. It is possible that disturbance of blood flow through basal ganglia associated with arterial malformations cause injury to the blood-brain barrier. A consequence of this may be precipitation and mineralization of serum proteins. Alkaline phosphatase (AP), which plays a role in cellular transport, may also be involved in pathogenesis of BSPDC; increase in AP activity in basal ganglia was observed in patients, while serum AP activity remained beyond normal values. Progressive inflammatory processes in the brain may also be responsible for calcifications [5]. Clinical symptoms of BSPDC are found in the literature as case reports, because the disease is very rare.
Corresponding author: Marlena Broncel, MD Department of Internal Diseases and Clinical Pharmacology Medical University of Lodz Kniaziewicza 1/5 Lodz 91-347, Poland Phone/fax: +48 426511059 E-mail: marlena.broncel @umed.lodz.pl 
The most common symptom of BSPDC is considered to be movement disorders, both Parkinsonism and hyperkinetic movement disorders (chorea, tremor, dystonia, athetosis, oro-facial dyskinesia). The second most common manifestation of BSPDC is cognitive impairment followed by cerebellar impairment and speech disorder. Psychiatric features (dementia, psychosis, affective disturbances), gait disorders, and sensory changes are also reported. Neurological disorders such as extrapyramidal symptoms, pyramidal symptoms, epileptic seizures, choreoathetosis, and glossopharyngeal neuralgia may also be associated with BSPDC. Diagnosis of BSPDC is based on finding pathognomonic basal ganglia calcifications in computed tomography or magnetic resonance imaging [6].

\section{Case report}

We would like to report the case of a 60-yearold patient with epileptic seizures, affective disturbances, only mild neurocognitive disorders and cardiomyopathy. A female patient was taken to the internal ward with a tentative diagnosis of recurrent syncope. On admission she gave a history of hypertension, worsening of exercise tolerance, and oedema of the lower extremities. The patient also reported depressive mood and memory deterioration. The patient reported that both physical and psychiatric symptoms had developed for the last twelve months. There was no history of alcohol abuse, nicotinism, previous surgery or family history of similar illness. On admission she was alert and cooperative. Physical examination of the respiratory system, heart and abdomen did not reveal anything specific. Bilateral oedema of the lower limbs was present. An ECG examination taken on admission revealed flattened T-waves in limb leads, biphasic T-waves in precordial leads V3-V5 and prolonged QTc interval (531 ms) (Figures 1A-D).

Laboratory results disclosed the following abnormalities: hypocalcaemia, hyperphospha-
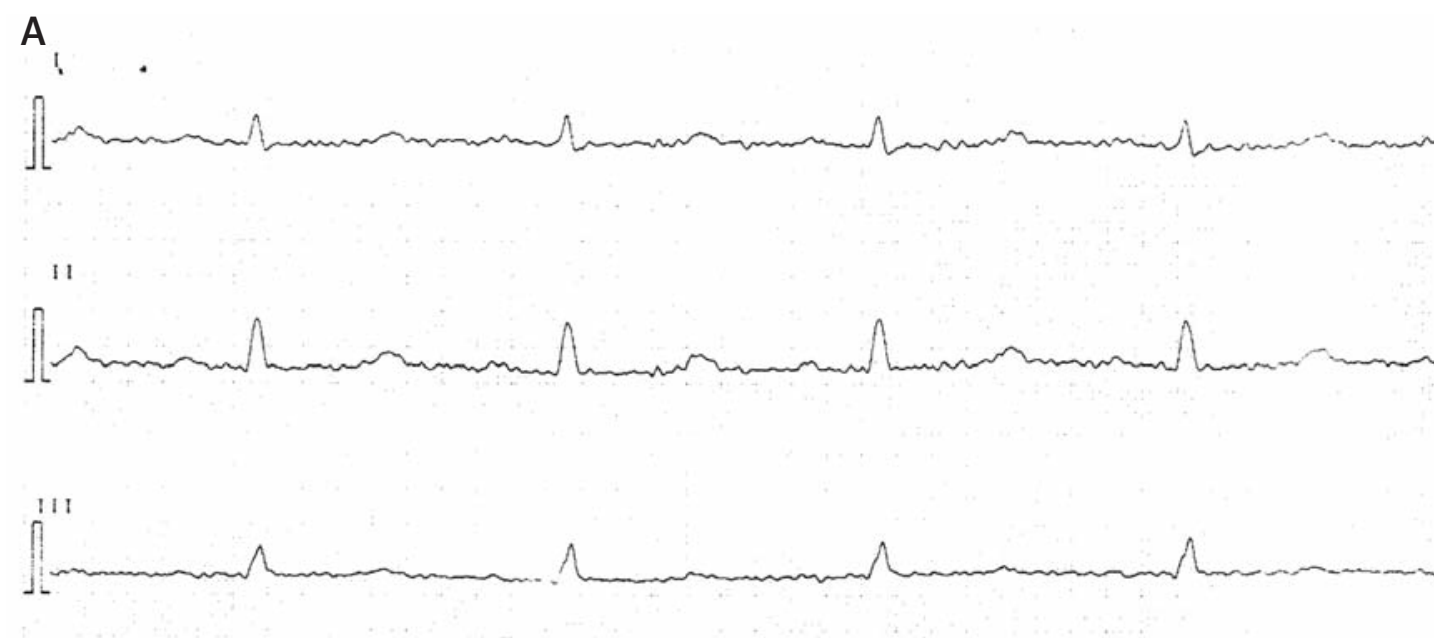

Data: 16.06 .08 Godzina: $12: 48: 39$

$\langle 50 \mathrm{~mm} / \mathrm{s} * 10 \mathrm{~mm} / \mathrm{mV} * 50 / 35 \mathrm{~Hz} * A s C A R D-32.00$ Aspel)

B

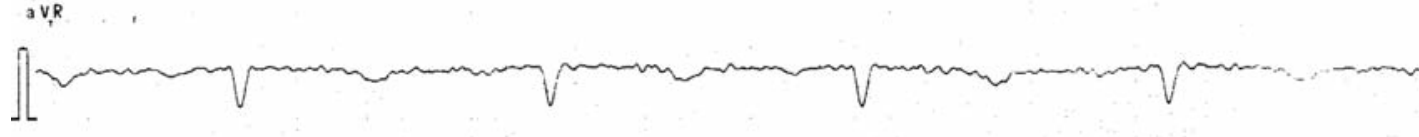

a VL
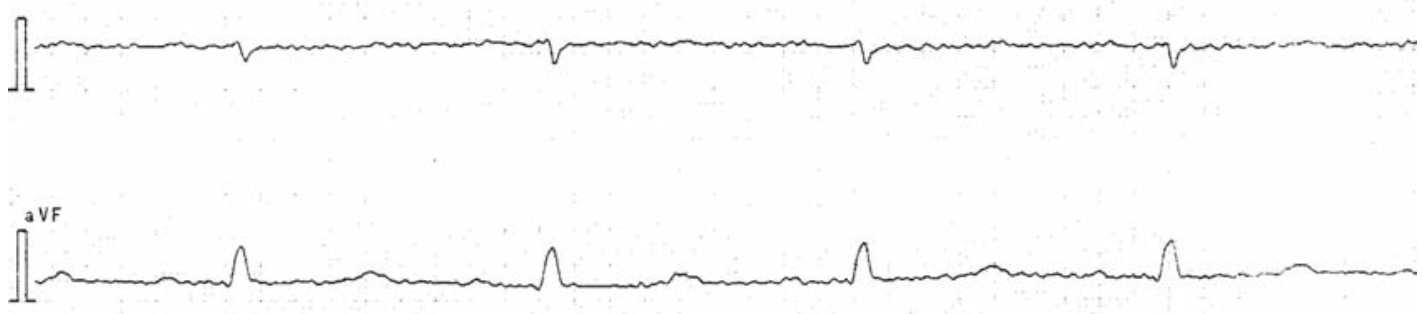

Figure 1A-B. ECG findings 


\section{C}

$$
\text { vi. . . }
$$

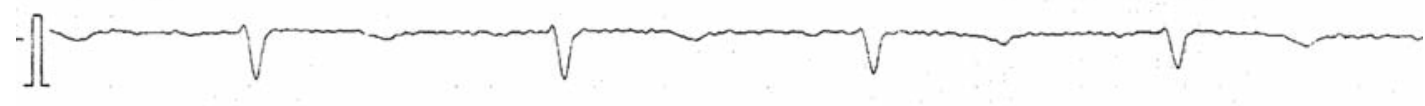

v 2
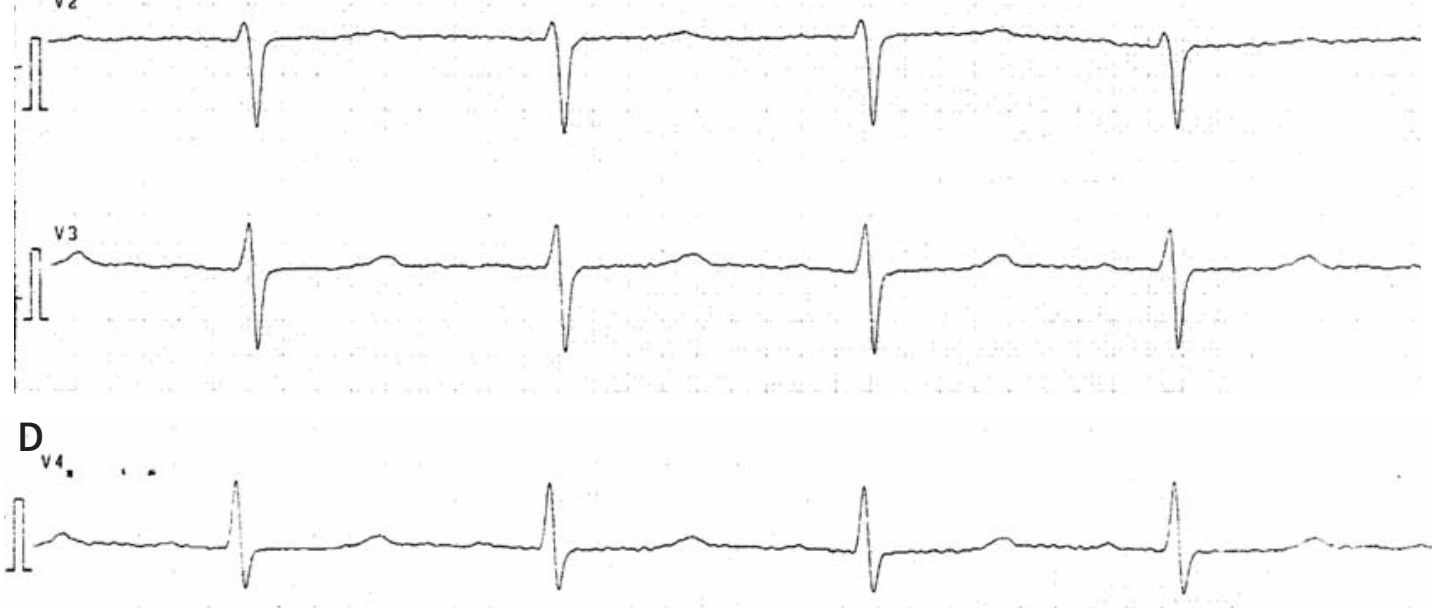

v 5
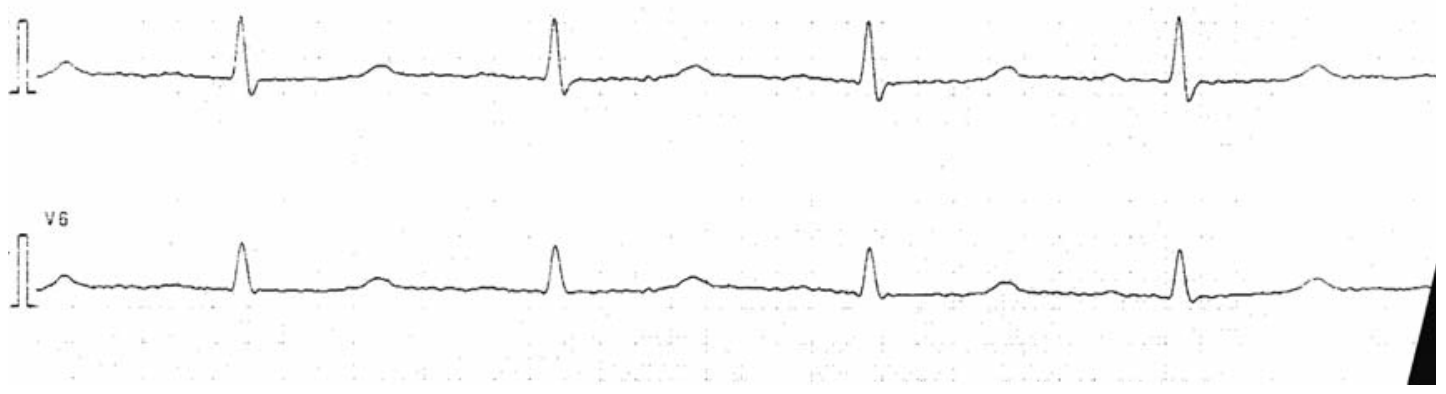

Figure 1 C-D. ECG findings

taemia, hypoparathyroidism, hypothyroidism, increased thyroid peroxidase antibodies and thyroglobulin antibodies (Table I). A transthoracic echocardiogram revealed generalized hypokinesis of the left ventricle and systolic dysfunction (reduced ejection fraction: $\mathrm{EF}=42 \%$ ). ECG Holter monitoring did not reveal any abnormalities which could be responsible for the recurrent syncope. No abnormalities of the ST segment typical for ischaemic heart disease were present in ECG Holter monitoring. Computed tomography (Figures 2-5) revealed bilateral, massive calcifications of the cerebellar dentate nucleus and white matter, lenticular nucleus: putamen and globus pallidus, caudate nucleus, thalamus, pons, white matter of centrum semiovale, grey matter of the fundus of fissures in the frontal lobe (superior frontal sulcus), parietal lobe (central sulcus), temporal lobe (superior temporal sulcus), and occipital lobe (calcarine sulcus and parieto-occipital fissure).

Electroencephalography did not show any abnormalities. Psychiatric examination revealed depression, anxiety and mild neurocognitive impairment (Mini-Mental State Examination: 23 points - MMSE) [7]. The patient reported depressive mood, apathy, and inability to make decisions, that had developed since one year ago. Finally she was too apathetic to perform daily living activities. Three

Table I. Laboratory abnormalities

\begin{tabular}{|lcc|}
\hline Serum parameter & Patient's result & Laboratory norm \\
\hline Calcium & $3.61 \mathrm{mg} / \mathrm{dl}$ & $8.5-10.8 \mathrm{mg} / \mathrm{dl}$ \\
\hline Phosphate & $7.3 \mathrm{mg} / \mathrm{dl}$ & $2.4-4.5 \mathrm{mg} / \mathrm{dl}$ \\
\hline Parathormone & $3.39 \mathrm{pg} / \mathrm{mol}$ & $10.0-70.0 \mathrm{pg} / \mathrm{mol}$ \\
\hline Thyrotropin & $12.39 \mu \mathrm{U} / \mathrm{ml}$ & $0.27-4.2 \mu \mathrm{U} / \mathrm{ml}$ \\
\hline Triiodothyronine & $3.38 \mathrm{pmol} / \mathrm{l}$ & $3.1-6.8 \mathrm{pmol} / \mathrm{l}$ \\
\hline Thyroxine & $8.52 \mathrm{pmol} / \mathrm{l}$ & $12.0-22.0 \mathrm{pmol} / \mathrm{l}$ \\
\hline $\begin{array}{l}\text { Thyroid peroxidase } \\
\text { antibodies (TPOAb) }\end{array}$ & $>600 \mathrm{U} / \mathrm{ml}$ & $0.0-34.0 \mathrm{U} / \mathrm{ml}$ \\
\hline $\begin{array}{l}\text { Thyroglobulin } \\
\text { antibodies (TgAb) }\end{array}$ & $600.8 \mathrm{IU} / \mathrm{ml}$ & $0.0-115.0 \mathrm{IU} / \mathrm{ml}$ \\
\hline
\end{tabular}




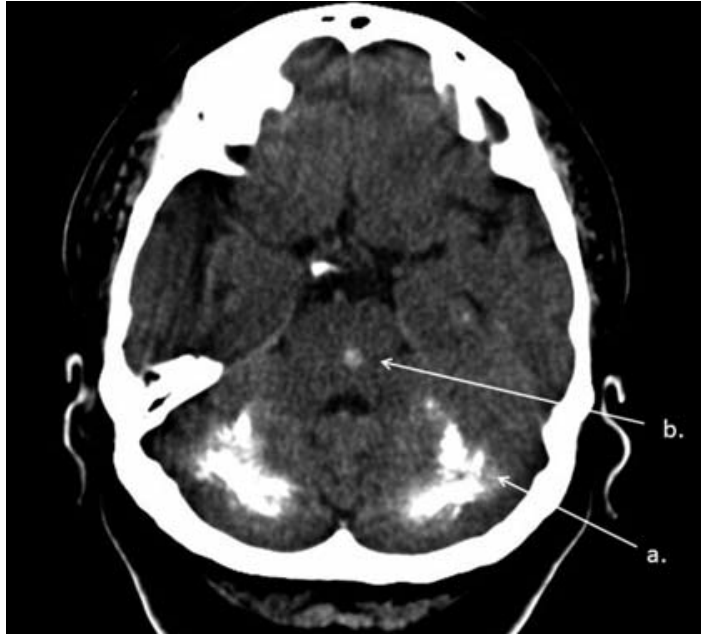

Figure 2. Brain CT. Bilateral massive calcifications of cerebellar dentate nucleus and white matter of cerebellum (a), calcification in pons (b)

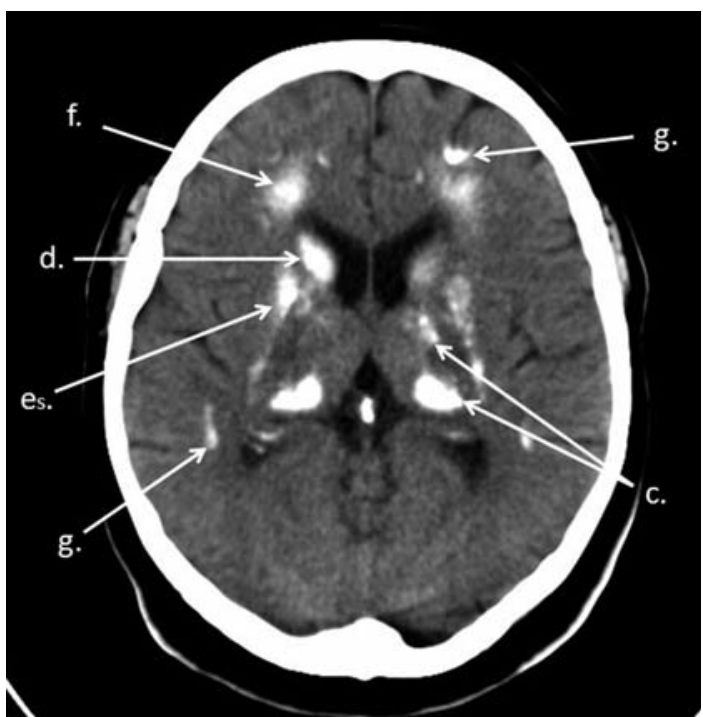

Figure 4. Brain CT. Calcifications of thalamus (c), caudate nucleus (d), putamen (es), white matter of centrum semiovale $(\mathrm{f})$, grey matter of brain fissures $(\mathrm{g})$

and ten days after admission episodes of unconsciousness were observed by medical professionals. Each episode lasted circa $15 \mathrm{~min}$; involuntary passing of urine was noted. The patient reported that episodes of unconsciousness had occurred about once a month during the last year. Each episode lasted 10-20 min. In some cases involuntary passing of urine was observed. Witnesses of these episodes related that after regaining consciousness, the patient's consciousness was clouded for about $30 \mathrm{~min}$. Typical epileptic seizures were not observed. ECG examination performed during an episode of unconsciousness did not reveal any abnormalities. Ultimately, the patient was diagnosed as having BSPDC and hypocalcaemic heart failure related to idiopathic hypoparathyroidism.

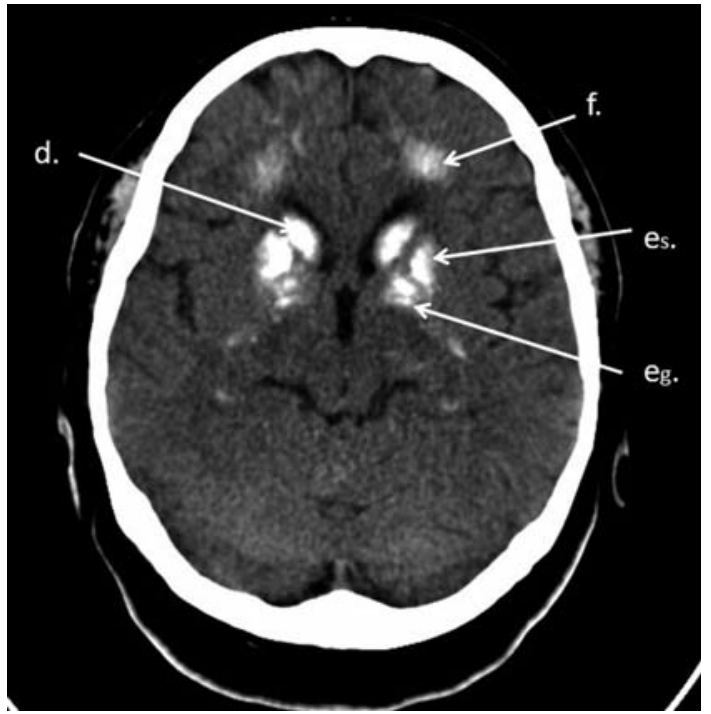

Figure 3. Brain CT. Calcifications of caudate nucleus (d), lenticular nucleus: putamen (es) and globus pallidus (eg), white matter of centrum semiovale ( $f$ )

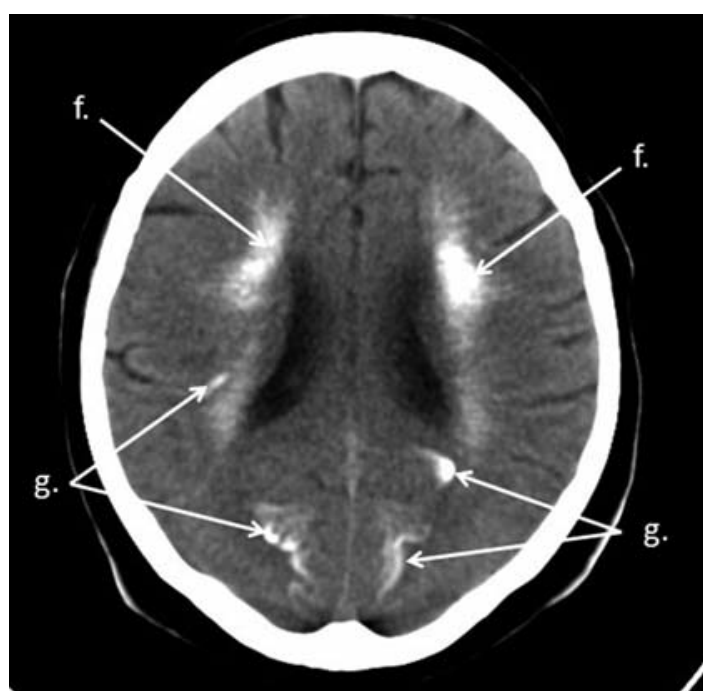

Figure 5. Brain CT. Calcifications of white matter of centrum semiovale $(\mathrm{f})$, grey matter of brain fissures $(\mathrm{g})$

Initially, the symptoms of hypocalcaemia were controlled with calcium gluconate infusion; subsequently oral calcium carbonate and $\alpha$-calcidol were started. Pharmacotherapy of heart failure with losartan, bisoprolol, spironolactone and hydrochlorothiazide was administered. An additional treatment with thyroxine, valproic acid and sertraline was also instituted. Restoration of normocalcaemia resulted in full clinical improvement of cardiac function in two weeks. Improvement in echocardiogram was also noted: signs of left ventricle hypokinesis retreated and EF increased up to $52 \%$. Attacks of unconsciousness responded well to valproic acid therapy.

Telephone contact with the patient's husband made three months after discharge from hospital 
confirmed that these attacks had not occurred, exercise tolerance had improved, and oedema of the lower extremities was not present. However, the patient's husband observed gradual deterioration of neurocognitive functions.

\section{Discussion}

In this case of BSPDC, attacks of unconsciousness were the predominant neurological manifestation. Our patient also presented hypocalcaemic cardiomyopathy. There are case reports presenting cases of reversible hypocalcaemic cardiomyopathy [8-11]. Long-lasting hypocalcaemia may result in cardiac dysfunction. Having in mind that laboratory results disclosed hypothyroidism (caused by autoimmune processes - antithyroid antibodies) it may be possible that this condition is partially responsible for cardiomyopathy. Hypothyroidism may cause both systolic and diastolic dysfunction. In our patient only systolic dysfunction was noted, which seems to be connected with calcium deficiency. Restoration of a normal calcium level resulted in clinical and echocardiographic improvement in two weeks. We conclude that two weeks time was too short a period to restore hormonal balance although thyroxine was added to the treatment regimen. The patient did not consent to coronary angiography, so we cannot rule out atherosclerosis and ischaemic heart disease. However, the patient did not give a history of retrosternal pain or any other symptoms that could suggest coronary artery disease. The laboratory findings and massive intracranial calcifications suggesting long-standing hypocalcaemia confirm our theory. Calcium is necessary for myocardial contractile function. Parathormone has a positive inotropic effect on the heart muscle that is mediated by increasing the entry of calcium into myocardial cells and the release of endogenous myocardial norepinephrine [12]. Myocardial sarcoplasmic reticulum is not able to sequestrate a sufficient quantity of calcium to initiate contraction. The strength of myocardial contraction is associated with extracellular calcium influx. It has also been proposed that a decrease of calcium levels in renal tubular cells may increase renal sodium reabsorption and via this mechanism promote sodium retention and the development of cardiac failure [10]. The role of calcium in the therapy of cardiac failure should be kept in mind as loop diuretics such as furosemide or torasemide may induce hypocalcaemia even in patients with normal parathormone levels. If congestive heart failure does not respond to conventional anti-failure therapy, hypocalcaemia should be taken into consideration. Therefore, the idea of calcium level determination in all patients with heart failure suggested in previous reports $[10,11]$ is supported by us. It is possible that patients with asymptomatic hypocalcaemia and heart failure treated with high doses of loop diuretics may develop severe hypocalcaemia and their state may deteriorate. Such a case has been previously reported [9].

In conclusion, patients with BSPDC can present a variety of symptoms. BSPDC cases are reported mainly by psychiatrists or neurologists. Our patient demonstrated only mild neurological and psychiatric symptoms but developed hypocalcaemic heart failure. Having in mind that cardiomyopathy associated with hypocalcaemia is reversible, we should determine the calcium level in all subjects with heart failure.

\section{Acknowledgments}

Sincere thanks to Bogdan Błaszczyk, MD and Marek Kazanek, MD for help with the description of CT scans.

\section{References}

1. Geschwind DH, Loginov M, Stern JM. Identification of a locus on chromosome $14 q$ for idiopathic basal ganglia calcification (Fahr disease). Am J Hum Genet 1990; 65: 764-72.

2. Kotan D, Aygul R. Familial Fahr disease in a Turkish Family. South Med J 2009; 102: 85-6.

3. Brodaty H, Mitchell P, Luscombe G, et al Familial idiopathic basal ganglia calcification (Fahr's disease) without neurological, cognitive and psychiatric symptoms is not linked to the IBGC1 locus on chromosome 14q. Hum Genet 2002; 110: 8-14.

4. Heymann-Szlachcinska A, Kisielewski J, Rybakowski J. Neurocognitive disorders in female patient with Fahr syndrome. Wiad Psych 2004; 7: 35-8.

5. Avrahami E, Cohn DF, Feibel M, Tadmor R. MRI demonstration and CT correlation of the brain in patients with idiopathic intracerebral calcification. J Neurol 1994; 241: 381-4.

6. Manyam BV. What is and what is not 'Fahr's disease'. Parkinsonism Relat Disord 2005; 11: 73-80.

7. Folstein MF, Folstein SE, McHugh PR. "Mini-mental state". A practical method for grading the cognitive state of patients for the clinician. J Psychiatr Res 1975; 12: 189-98.

8. Altunbaș H, Balcı MK, Yazıcıoğlu G, Semiz E, Özbilim G, Karayalçın Ü. Hypocalcemic cardiomyopathy due to untreated hypoparathyroidism. Horm Res 2003; 59: 201-4.

9. Avsar A, Dogan A, Tavli T. A rare cause of reversible dilated cardiomyopathy: hypocalcemia. Echocardiography 2004; 21: 609-12.

10. Chavan CB, Sharada K, Rao HB, Narsimhan C. Hypocalcemia as a cause of reversible cardiomyopathy with ventricular tachycardia. Ann Intern Med 2007; 146: 541-2.

11. Tziomalos K, Kakavas N, Kountana E, Harsoulis F, Basayannis E. Reversible dilated hypocalcaemic cardiomyopathy in a patient with primary hypoparathyroidism. Clin Endocrinol 2006; 64: 717-8.

12. Bogin E, Harary I. The relationship of calcium and parathyroid hormone in their effect on heart cells. Mol Cell Biochem 1987; 77: 29-35. 